\title{
L'importanza del vaccino anti Covid-19 nei pazienti affetti da malattia del rene policistico autosomico dominante dell'adulto (ADPKD)
}

\author{
Maria Teresa Sciarrone Alibrandi ${ }^{1}$, Giancarlo Joli ${ }^{1}$, Rodolfo F. Rivera ${ }^{2}$, Elena Brioni ${ }^{1}$, Romina Bucci ${ }^{1}$, Marta Vespa $^{1}$ \\ ${ }^{1}$ University Vita Salute San Raffaele, IRCCS San Raffaele Scientific Institute, Milano - Italy \\ ${ }^{2}$ U.O. Nefrologia e Dialisi, Ospedale di Desio - ASST - Brianza, Desio - Italy
}

\begin{abstract}
The importance of the Covid-19 vaccine in patients suffering from adult autosomal dominant polycystic kidney disease (ADPKD)

The SARS-CoV-2 (Covid-19) infection affected about 106 million people worldwide and the total amount of casualties now sits at a staggering 2 millions.

Chronic Kidney Disease (CKD) emerged as the first risk factor in worst patients, not considering old age. Kidney disease and acute kidney injury have been correlated with a higher chance of death. This combination of CKD and higher Covid-19 related mortality requires immediate response from a prevention point of view at first and then from a therapeutic one.

There is not a clear relation between Covid-19 and ADPKD. What can be inferred is the following: Covid uses the ACE2 receptors on cell membranes to "lock on" its target. It is well-established in fact that the RAAS is more active in ADPKD patients and it may represent an additional risk factor for these patients.

At the moment three Covid-19 vaccines have been approved, and two of them have been already administered, such as Pfizer BioNTech and Moderna, sharing the same mechanism. AstraZeneca released a third option. All of them are completely safe and reliable, each one with its own feature.

Therefore, considering how delicate ADPKD patients are, vaccination is strongly recommended.
\end{abstract}

Keywords: ADPKD patients, SARS-CoV-2 (Covid-19), Vaccine

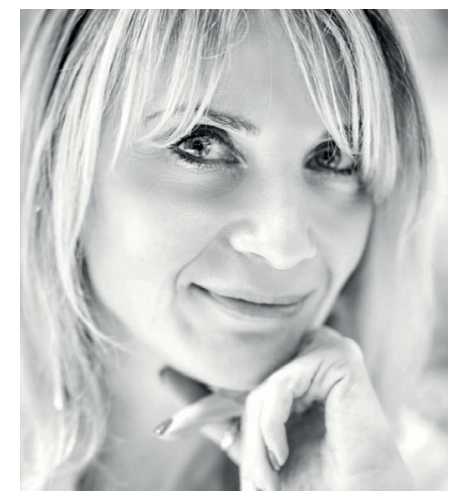

L'infezione da SARSCoV-2 (Covid-19) ha colpito, nel corso della pandemia, circa 106 milioni di persone nel mondo e si contano, a tutt'oggi, circa 2 milioni di decessi. Una caratteristica ancora enigmatica di tale infezione è l'ampia gamma di manifestazioni cliniche che variano dalla pressoché totale assenza di sintomi

Received: March 4, 2021

Accepted: March 6, 2021

Published online: May 22, 2021

Indirizzo per la corrispondenza:

Maria Teresa Sciarrone Alibrandi

University Vita Salute San Raffaele

IRCCS San Raffaele Scientific Institute

Via Olgettina 60

20132 Milano - Italy

sciarronealibrandi.mariateresa@hsr.it a forme estremamente gravi, con compromissione multiorgano dall'esito inesorabilmente fatale. L'elevata frequenza di infezioni asintomatiche, inoltre, ha indubbiamente contribuito alla rapida diffusione mondiale della SARS-CoV-2. Risulta, pertanto, di fondamentale importanza individuare precocemente le categorie di soggetti fragili, più a rischio di sviluppare una forma grave di malattia. A tutt'oggi è noto come l'età rappresenti il principale fattore di rischio in termini di mortalità, ma, certamente, non è il solo (1).

Sicuramente, alcune patologie concomitanti predispongono con un'elevata probabilità a sviluppare una forma di malattia più grave e spesso mortale.

Le prime segnalazioni nel gennaio 2020 evidenziavano solo diabete, ipertensione e malattie cardiovascolari come importanti fattori di rischio (1). Solo più recentemente, la malattia renale cronica (CKD) è emersa come il fattore di rischio più comune, dopo l'età avanzata, per le forme più serie di infezione e per aumentato rischio di mortalità, sino a tre volte maggiore rispetto alla popolazione generale.

È noto che i pazienti affetti da CKD generalmente presentano, di per sé, un'aumentata mortalità rispetto alla popolazione generale, a causa di problemi correlati all'aumentato 
rischio di sviluppare malattie cardiovascolari e tumorali, ma anche, sicuramente, alla maggiore incidenza di complicanze infettive che contribuiscono in maniera decisiva alla ridotta aspettativa di vita $(2,3)$. Verosimilmente, lo stress fisiologico causato dalla risposta infiammatoria a un processo infettivo potrebbe ulteriormente indebolire organi già deficitari a causa della malattia cronica.

Nel caso di SARS-CoV-2, peraltro, questa ipotesi risulta ulteriormente coerente con il fatto che rene e il cuore presentano la più alta espressione di recettori ACE2, con i quali, appunto, è stato dimostrato il legame del virus (4).

Molteplici recenti studi condotti in ambienti intensivi su pazienti sottoposti a ventilazione invasiva hanno, infatti, confermato che il danno renale acuto (AKI) e la necessità di terapia renale sostitutiva (CRRT) in corso di Covid-19 rappresentano un fattore di rischio di aumentata mortalità e che questo fattore di rischio è nettamente più elevato in presenza di malattia renale preesistente, definendo l'insufficienza renale come fattore prognostico assolutamente sfavorevole per lo sviluppo di infezione grave a esito infausto $(5,6)$.

Da ultimo, va segnalato che gli effetti dell'epidemia sui pazienti già sottoposti a dialisi, secondo quanto evidenziato e riportato da un'indagine dalla Società Italiana di Nefrologia (7), sono stati decisamente importanti. Fra i dializzati, si è, infatti, registrata una mortalità dieci volte superiore a quella a oggi stimata nella popolazione generale, soprattutto durante la seconda fase della pandemia ( $26 \%$ vs $2,4 \%$ ).

Pertanto, i pazienti affetti da qualsivoglia nefropatia rappresentano realmente una coorte di soggetti estremamente a rischio a cui è necessario prestare la massima attenzione, adottando ogni sforzo per prevenire la progressione della malattia al fine di ridurne la mortalità.

I pazienti affetti da malattia del rene policistico autosomico dominante dell'adulto (ADPKD), essendo affetti da una sofferenza renale cronica che spesso condiziona un'insufficienza renale in diversi stadi di gravità, fanno, quindi, parte a pieno titolo di questa categoria a rischio.

Come precedentemente descritto, è noto che SARS-CoV-2 utilizza il recettore ACE2 per infettare le cellule, che è sito di legame per uno degli enzimi centrali nel sistema reninaangiotensina-aldosterone (RAAS) (4).

Questo meccanismo, quindi, sottintende il processo patogenetico dell'infezione e il conseguente danno d'organo.

È noto, inoltre, a questo proposito, che proprio i pazienti affetti da ADPKD hanno, di per sé, un'iperattivazione del RAAS secondaria alla nefropatia di base anche in stadi relativamente precoci della malattia, mentre non è stata ancora studiata la presenza di una maggiore o minore espressione del recettore ACE2 a livello tissutale (8). Questo potrebbe, quindi, essere un ulteriore fattore predisponente allo sviluppo di forme più serie di infezione in corso di Covid-19, al di là dell'insufficienza renale che, già di per sé, rappresenta un fattore di rischio.
L'elevata prevalenza di CKD nei pazienti ADPKD richiede, quindi, un'azione urgente di tipo preventivo, prima ancora che terapeutico.

I vaccini rappresentano da sempre una delle principali armi che l'umanità ha per combattere le malattie infettive.

In soli undici mesi, grazie a tutte le risorse di conoscenza disponibili, è stato ottenuto un vaccino, e questo rappresenta una conquista inestimabile nel corso di una pandemia.

Tutti i vaccini attualmente approvati e anche quelli ancora in studio sono stati messi a punto per indurre una risposta immunitaria che blocchi la proteina Spike (proteina di superficie del SARS-CoV-2) e che, quindi, impedisca l'infezione delle cellule $(9,10)$.

Il vaccino Covid-19 mRNA BNT162b2 (Comirnaty), noto come Pfizer-BioNTech, è stato il primo vaccino disponibile in Italia per prevenire il Covid-19, nei soggetti a partire dai 16 anni di età.

Il vaccino è stato autorizzato da EMA (European Medicines Agency, Agenzia Europea per i Medicinali) (11) e AIFA (Agenzia Italiana del Farmaco) $(12,13)$. Per la sua realizzazione sono state regolarmente rispettate tutte le consuete fasi di verifica in merito all'efficacia e alla sicurezza. In Italia, la sua somministrazione ha avuto inizio il 27 dicembre, secondo il piano nazionale di vaccinazione che prevede più fasi. II vaccino Covid-19 mRNA BNT162b2 (Comirnaty) è stato il primo vaccino ad arrivare in Italia, seguito da Covid-19 mRNA-1273, più comunemente noto come vaccino Moderna con il medesimo meccanismo d'azione, approvato da EMA il 6 gennaio 2021 (14) e da AIFA il giorno successivo.

Come funzionano il vaccino Pfizer BioNTech e quello Moderna?

Prima di addentrarsi nel meccanismo d'azione del vaccino è necessario ricordare come agisce il virus SARS-CoV-2.

II vaccino Covid-19 mRNA BNT162b2 (Comirnaty), così come il Covid-19 Vaccine Moderna, contiene le molecole di RNA messaggero (mRNA) che presentano al loro interno le indicazioni per costruire le proteine Spike del virus SARSCoV-2. Nel vaccino, le molecole di mRNA sono inserite in una vescicola lipidica, che protegge I'mRNA per evitarne la degradazione e per impedire che I'mRNA venga immediatamente distrutto dalle difese immunitarie in quanto riconosciuto come estraneo dalle difese immunitarie dell'organismo $(9,10,15)$.

Una volta iniettato il vaccino, I'mRNA viene assorbito nel citoplasma delle cellule e avvia la sintesi delle proteine Spike. La loro presenza stimola, così, la produzione, da parte del sistema immunitario, di anticorpi specifici.

Una volta svolta l'azione di induzione anticorpale, I'mRNA del vaccino si degrada naturalmente nell'arco di pochi giorni. Non esiste, pertanto, alcun rischio che venga integrato nel DNA delle cellule dell'organismo in via definitiva $(9,10,15)$.

Il vaccino Pfizer BioNTech è sicuro e, in merito alla sua sicurezza e alla sua qualità, si è espresso anche il New England Journal of Medicine (10). 
Il dubbio mediaticamente espresso su una troppo rapida produzione e distribuzione di questo vaccino può essere facilmente fugato. Gli studi sui vaccini anti Covid-19, compreso il vaccino Covid-19 mRNA BNT162b2 (Comirnaty), sono iniziati nella primavera del 2020 e, indubbiamente, sono durati pochi mesi rispetto ai tempi abituali. Hanno, però, beneficiato della partecipazione di un numero molto più elevato di soggetti: un numero dieci volte superiore rispetto agli standard degli studi analoghi per lo sviluppo dei vaccini. È stato, pertanto, messo a punto uno studio di grandi dimensioni, sufficienti per dimostrare efficacia e sicurezza del vaccino, e non è stata saltata nessuna fase sperimentale normalmente prevista (15).

In termini di efficacia, gli studi effettuati l'hanno attestata pari al 95\%.

Un altro tipo di vaccino anti SARS-CoV-2 che sarà a breve disponibile è il vaccino Covid-19 AstraZeneca, approvato da EMA il 29 gennaio 2021 (16) e da AIFA il giorno dopo (13). Questo è un vaccino destinato a prevenire la malattia da coronavirus nelle persone di età pari o superiore ai 18 anni ed è progettato per preparare il sistema immunitario a identificare e contrastare il SARS-CoV-2 (17).

II meccanismo, però, è sostanzialmente differente rispetto al precedente.

Il vaccino è composto, infatti, da un adenovirus di scimpanzé incapace di replicarsi (ChAdOx1, Chimpanzee Adenovirus Oxford 1) e modificato per veicolare l'informazione genetica destinata a produrre la proteina Spike del virus SARS-CoV-2.

In sintesi, l'adenovirus è stato geneticamente modificato. Una delle proteine dell'adenovirus è stata rimossa e sostituita con la proteina Spike del SARS-CoV-2, verso cui si genererà la risposta immunitaria nell'organismo ospite. Qual è il potenziale vantaggio di questo approccio rispetto a quello usato per gli altri vaccini? La "scatola del vaccino" è incredibilmente resistente, in quanto rappresentata da un altro agente virale. Mentre gli altri vaccini richiedono per la loro conservazione temperature molto basse, questo tipo di vaccino può essere tranquillamente conservato sei mesi in un comune frigorifero (17).

Questo vaccino è sicuro ed efficace al pari di quello precedentemente descritto? A questo quesito rispondono gli studi clinici. I dati di sicurezza sono risultati molto buoni. Per quanto riguarda il profilo di efficacia, i dati sinora disponibili l'hanno attestata pari a circa il $60 \%$. Benché, in termini puramente numerici, l'efficacia sembri, quindi, significativamente minore rispetto al vaccino Covid-19 mRNA BNT162b2, in realtà, il vaccino AstraZeneca presenta una buona efficacia, sicuramente superiore, per esempio, a quella del comune vaccino antinfluenzale impiegato annualmente e che ogni anno fornisce, comunque, un'ottima copertura contro l'influenza stagionale, in special modo nei soggetti fragili (18).

L'AIFA ha autorizzato I'utilizzo di questo vaccino sino ai 55 anni di età. Non sono ancora disponibili dati sufficienti per stabilire il grado di efficacia del vaccino nella popolazione oltre questo limite d'età. Tuttavia ci si aspetta un'adeguata protezione, sia sulla base dell'esperienza ottenuta con altri vaccini sia sulla base della buona risposta immunitaria osservata in questa fascia d'età.

In sintesi, allo stato attuale delle conoscenze e delle risorse disponibili, la Commissione tecnico-scientifica dell'AIFA ha suggerito per la vigente campagna vaccinale e per il prossimo futuro un utilizzo preferenziale (13):

- dei vaccini a mRNA nei soggetti anziani e/o a più alto rischio di sviluppare una malattia grave;

- del vaccino AstraZeneca nei soggetti tra i 18 e i 55 anni. Sulla base dei risultati di immunogenicità e dei dati di sicurezza, il rapporto beneficio/rischio del vaccino risulta comunque favorevole anche nei soggetti di età più avanzata che non presentino specifici fattori di rischio.

Oltre a quelli approvati e in utilizzo, esistono, comunque, decine di vaccini in sperimentazione clinica e preclinica i cui risultati preliminari sono molto promettenti.

I grandi numeri evidenziano un'efficacia vaccinale per SARS-CoV-2 e un profilo di sicurezza comunque molto alti per tutti i vaccini sinora in esame (18).

Purtroppo, a livello mediatico, ci sono ancora molta confusione e notizie fuorvianti al riguardo, e questo può far perdere di vista l'importanza di vaccinarsi, soprattutto per le categorie a rischio quali i pazienti affetti da malattia del rene policistico, soprattutto se anziani e ipertesi.

La storia insegna da sempre che vaccinarsi significa vivere e non esiste malattia che sia meglio contrarre piuttosto che vaccinarsi, perché, comunque, esiste sempre un rischio maggiore rispetto a quello vaccinale, in particolar modo per i soggetti più vulnerabili.

A questo proposito va segnalato lo sforzo dimostrato dalla Società Italiana di Nefrologia e dalle Associazioni di pazienti (ANED) e di malattie renali (FIR) a cui è seguita una lettera di impegno da parte del Commissario Straordinario per l'emergenza Covid-19, Dottor Domenico Arcuri, relativa a un aggiornamento tempestivo del piano strategico riguardo ai pazienti fragili e alla loro priorità vaccinale.

L'elevato rischio di infezione grave e di aumentata mortalità in presenza di insufficienza renale, ormai documentato da plurime evidenze cliniche, rende, infatti, il vaccino altamente raccomandato per questa coorte di pazienti, anche indipendentemente dal fattore età, al fine di ridurne in maniera drastica il rischio di ospedalizzazione e di mortalità.

In tal senso, è opportuno evidenziare il tenace impegno della Società Italiana di Nefrologia, volto a richiedere la revisione del piano nazionale strategico per la vaccinazione da parte del Ministero della Salute, dell'AIFA, del Comitato tecnico-scientifico e del Commissario Straordinario per l'emergenza Covid-19. Tale richiesta, decisamente sentita e supportata anche da altre associazioni di settore, come ANED (Associazione Nazionale Emodializzati Dialisi e Trapianto,) 
e FIR: (Fondazione Italiana del Rene), è stata recentemente soddisfatta, con l'inclusione di tutti i pazienti con patologia renale, dializzati e portatori di trapianto renale nelle categorie di persone vulnerabili e con priorità per la vaccinazione, come annunciato nella lettera di impegno del 9 febbraio firmata dal Commissario Domenico Arcuri.

I pazienti nefropatici, dializzati e trapiantati hanno pagato un tributo molto alto alla pandemia SARS-CoV-2. Per tale motivo, è essenziale che tale popolazione particolarmente fragile, come quella dei pazienti policistici renali, anch'essi esposti quantomeno a un rischio di analoga entità, sia non soltanto protetta nel minor tempo possibile, ma anche monitorata nel tempo, per rilevare tempestivamente gli effetti immunologici che seguiranno alla vaccinazione.

\section{Disclosures}

Conflict of interest: The authors declare no conflict of interest. Financial support: This research received no specific grant from any funding agency in the public, commercial, or not-for-profit sectors.

\section{Bibliografia}

1. Rashedi J, Mahdavi Poor B, Asgharzadeh V, et al. Risk Factors for COVID-19. Infez Med. 2020;28(4):469-474. PubMed

2. Adapa S, Chenna A, Balla M, et al. COVID-19 pandemic causing acute kidney injury and impact on patients with chronic kidney disease and renal transplantation. J Clin Med Res. 2020;12(6):352-361. Crossref PubMed

3. Gagliardi I, Patella G, Michael A, Serra R, Provenzano M, Andreucci M. COVID-19 and the kidney: from epidemiology to clinical practice. J Clin Med. 2020;9(8):2506. Online Crossref PubMed

4. Hamming I, Timens W, Bulthuis MLC, Lely AT, Navis G, van Goor $\mathrm{H}$. Tissue distribution of ACE2 protein, the functional receptor for SARS coronavirus. A first step in understanding SARS pathogenesis. J Pathol. 2004;203(2):631-637. Crossref PubMed

5. Stephens JR, Stümpfle R, Patel P, et al. Analysis of Critical Care Severity of IIIness Scoring Systems in Patients With Coronavirus
Disease 2019: A Retrospective Analysis of Three U.K. ICUs. Crit Care Med. 2021;49(1):e105-e107. Crossref PubMed

6. Fominskiy EV, Scandroglio AM, Monti G, et al; COVID-BioB Study Group. Prevalence, Characteristics, Risk Factors, and Outcomes of Invasively Ventilated COVID-19 Patients with Acute Kidney Injury and Renal Replacement Therapy. Blood Purif. 2021;50(1):102-109. Crossref PubMed

7. Quintaliani G, Reboldi G, Di Napoli A, et al; Italian Society of Nephrology COVID-19 Research Group. Exposure to novel coronavirus in patients on renal replacement therapy during the exponential phase of COVID-19 pandemic: survey of the Italian Society of Nephrology. J Nephrol. 2020;33(4):725-736. Crossref PubMed

8. Sciarrone Alibrandi MT, Vespa M. Kidney, ADPKD and Covid-19: the double role of renal fragility. G Clin Nefrol E Dialisi. 2020;32(1):99-101. Crossref

9. Polack FP, Thomas SJ, Kitchin N, et al; C4591001 Clinical Trial Group. Safety and Efficacy of the BNT162b2 mRNA Covid-19 Vaccine. N Engl J Med. 2020;383(27):2603-2615. Crossref PubMed

10. Anderson EJ, Rouphael NG, Widge AT, et al; mRNA-1273 Study Group. Safety and Immunogenicity of SARS-CoV-2 mRNA-1273 Vaccine in Older Adults. N Engl J Med. 2020;383(25):24272438. Crossref PubMed

11. Glanville D. COVID-19 vaccines: key facts. European Medicines Agency. 2020. Online

12. Vaccini a mRNA | Agenzia Italiana del Farmaco. Online

13. Vaccini a vettore virale | Agenzia Italiana del Farmaco. Online

14. Glanville D. EMA recommends COVID-19 Vaccine Moderna for authorisation in the EU. European Medicines Agency. 2021. Online

15. Sharma O, Sultan AA, Ding $H$, Triggle CR. A review of the progress and challenges of developing a vaccine for Covid-19. Front Immunol. 2020;11:585354. Online

16. Pinho AC. EMA recommends COVID-19 Vaccine AstraZeneca for authorisation in the EU. European Medicines Agency. 2021. Online

17. Knoll MD, Wonodi C. Oxford-AstraZeneca COVID-19 vaccine efficacy. Lancet. 2021;397(10269):72-74. Crossref PubMed

18. Voysey M, Clemens SAC, Madhi SA, et al; Oxford COVID Vaccine Trial Group. Safety and efficacy of the ChAdOx1 nCoV-19 vaccine (AZD1222) against SARS-CoV-2: an interim analysis of four randomised controlled trials in Brazil, South Africa, and the UK. Lancet. 2021;397(10269):99-111. Crossref PubMed 